\title{
CONTINUITY OF LINEAR OPERATORS COMMUTING WITH CONTINUOUS LINEAR OPERATORS
}

\author{
BY \\ B. E. JOHNSON
}

1. Introduction. In [6] and [8] we showed that centralisers (that is operators commuting with multiplication) on certain Banach algebras are necessarily continuous. In the present paper we consider whether an operator on a Banach space commuting with a continuous or a closed operator must itself be continuous. We also consider the more general problem of whether an operator $S ; E_{1} \rightarrow E_{2}$, satisfying $S T_{1}=T_{2} S$, with $T_{1}, T_{2}$ continuous, must be continuous. If the operators $T_{1}, T_{2}$ are operators of scalar type [3, p. 226] or operators in $B K$ spaces [15, p. 29] given by triangular matrices, we see that the only discontinuous $S$ are the obvious ones. In $\S 8$ we consider operators in $L_{1}(-\infty,+\infty)$ and $\mathscr{C}(-\infty,+\infty)$ commuting with the translation operator $(T f)(x)=f(x+1)$. For the $L_{1}$ space any operator commuting with $T$ is continuous, whereas in $\mathscr{C}(-\infty,+\infty)$ we can find a discontinuous operator commuting with all the translation operators.

A full solution to this problem might be useful in discussing the extent to which discontinuous homomorphisms between Banach algebras exist since if $\phi$ is a homomorphism it satisfies $\phi(a b)=\phi(a) \phi(b)$ which can be written $\phi L_{a}=L_{\phi(a)} \cdot \phi$ where $L_{a} x=a x$.

2. Discontinuous commutants. The Banach-Steinhaus theorem [2, p. 27] makes it necessary to employ transfinite methods to "construct" discontinuous operators in Banach spaces. Thus it can be difficult, when considering whether there are discontinuous operators satisfying certain conditions, to show that the answer is affirmative. We consider two cases in which the construction is simple. First, however, some notation.

Throughout the paper $\mathscr{B}(E, F)$ will denote the space of continuous linear transformations from $E$ into $F$. We write $\mathscr{B}(E)=\mathscr{B}(E, E)$. For any linear transformation $S, \mathscr{R}(S), \mathscr{N}(S), \sigma(S)$ and $\sigma_{p}(S)$ will denote the range, null space, spectrum and point spectrum of $S$ respectively. Transformations are not necessarily continuous unless specifically stated to be so. Similarly, when we speak of the codimension of a linear subspace $L$ of a vector space $E$ we mean the number of elements of a Hamel basis in $E / L$. Finally, all vector spaces will be over $C$, the field of complex numbers, unless (in part of $\S 8$ ) specifically stated to be otherwise.

2.1. LEMMA. Let $E_{1}, E_{2}$ be two topological vector spaces, $T_{1} \in \mathscr{B}\left(E_{1}\right), T_{2} \in \mathscr{B}\left(E_{2}\right)$ and $\lambda \in \sigma_{p}\left(T_{2}\right)$ such that $\mathscr{R}\left(T_{1}-\lambda I_{1}\right)$ is not closed in $E_{1}$. Then there is a discontinuous linear map $S ; E_{1} \rightarrow E_{2}$, with $S T_{1}=T_{2} S$.

Received by the editors October 1, 1965 . 
Proof. Let $S$ be a linear transformation on $E_{1}$ into $\mathscr{N}\left(T_{2}-\lambda I_{2}\right)\left(I_{1}, I_{2}\right.$ are the identity operators in $\left.E_{1}, E_{2}\right)$ which is zero on $\mathscr{R}\left(T_{1}-\lambda I_{1}\right)$ but not on its closure. Such a transformation can easily be constructed using a Hamel basis. Then $S\left(T_{1}-\lambda I_{1}\right)=0=\left(T_{2}-\lambda I_{2}\right) S$ and so $S T_{1}=\lambda S=T_{2} S$.

2.2. RemarK. A similar construction will give the same result if we assume $\mathscr{R}\left(T_{1}-\lambda I_{1}\right)$ closed and of infinite codimension, unless every linear transformation of $E_{1} / \mathscr{R}\left(T_{1}-\lambda I_{1}\right)$ into $\mathscr{N}\left(T_{2}-\lambda I_{2}\right)$ is continuous. This would certainly not be the case if $E_{1}$ were a metrisable locally convex space.

The following will make the statement of some of our theorems easier.

2.3. Lemma. Let $E, F$ be two Fréchet spaces, $T \in \mathscr{B}(E, F)$ with $\mathscr{R}(T)$ of finite codimension. Then $\mathscr{R}(T)$ is closed.

This result for Banach spaces is Lemma 332 on p. 275 of [9]. The proof for Fréchet spaces is the same.

We conclude with two extremely useful results. $T_{1}, T_{2}$ are linear operators in the vector spaces $E_{1}, E_{2}$ and $S$ is a linear operator $E_{1} \rightarrow E_{2}$ with $S T_{1}=T_{2} S$. We shall say that $S$ commutes with $\left\{T_{1}, T_{2}\right\}$.

2.4. LeMmA. If $R$ is a rational function regular on $\sigma\left(T_{1}\right)$ and $\sigma\left(T_{2}\right)$ then $\operatorname{SR}\left(T_{1}\right)$ $=R\left(T_{2}\right) S$.

2.5. LEMMA.

(i) $S \mathscr{N}\left(T_{1}\right) \subset \mathscr{N}\left(T_{2}\right)$.

(ii) $S \mathscr{R}\left(T_{1}\right) \subset \mathscr{R}\left(T_{2}\right)$.

(iii) If $S$ is one-to-one then $\sigma_{p}\left(T_{1}\right) \subset \sigma_{p}\left(T_{2}\right)$.

3. Operators commuting with normal operators. Throughout this section and the next $T, T_{1}, T_{2}$ are bounded normal operators in the Hilbert spaces $H, H_{1}, H_{2}$ respectively and $S$ is a linear operator from $H_{1}$ into $H_{2}$ commuting with $\left\{T_{1}, T_{2}\right\}$. $E(\lambda), E_{1}(\lambda), E_{2}(\lambda)$ are the resolutions of the identity for $T, T_{1}, T_{2}$ (see [4, p. 889]; we shall use results in Chapter $X$ of [4] without further reference).

3.1. Lemma. Let $A$ be an index set, $L$ a linear map $H_{1} \rightarrow H_{2}$ and, for each $\alpha \in A$, $P_{\alpha}$ an orthogonal projection in $H_{2}$ with $P_{\alpha} L$ continuous. Let $N=\bigcap \mathscr{N}\left(P_{\alpha}\right)(\alpha \in A)$ and let $P$ be the orthogonal projection onto $N^{\perp}$. Then $P L$ is continuous.

Proof. If $x_{n} \rightarrow 0$ in $H_{1}, P L x_{n} \rightarrow y$ in $H_{2}$ then, since $P, P_{\alpha}$ are projections with $\mathscr{N}(P) \subset \mathscr{N}\left(P_{\alpha}\right)$, we have $P_{\alpha} P=P_{\alpha}$ and so $P_{\alpha} L x_{n}=P_{\alpha} P L x_{n} \rightarrow P_{\alpha} y$. But as $P_{\alpha} L$ is continuous this implies $P_{\alpha} y=0$ for all $\alpha \in A$, that is $y \in N$ and $P y=0$. However $y=\lim _{n} P L x_{n}=\lim _{n} P\left(P L x_{n}\right)=P y=0$ and so $P L$ is continuous by the closed graph theorem.

3.2. Theorem. Let $G$ be an open set in $C$. Then

$$
\bigcap_{\lambda \in G} \mathscr{R}\left[(T-\lambda I)^{2}\right]=\mathscr{N}(E(G))=\mathscr{R}(I-E(G)) .
$$


Proof. (i) $\xi \in \bigcap_{\lambda_{0} \in G} \mathscr{R}\left[\left(T-\lambda_{0} I\right)^{2}\right]$ if and only if $\left(\lambda-\lambda_{0}\right)^{-2} \in L_{1}(\mu)$ for all $\lambda_{0} \in G$, where $\mu(X)=E(X) \xi$.

Suppose $\left(T-\lambda_{0} I\right)^{2} \eta=\xi$ and put $\nu(X)=E(X) \eta$. Then

$$
\begin{aligned}
\mu(X) & =E(X) \int_{C}\left(\lambda-\lambda_{0}\right)^{2} E(d \lambda) \eta \\
& =\int_{X}\left(\lambda-\lambda_{0}\right)^{2} \nu(d \lambda),
\end{aligned}
$$

so that $\mu=\left(\lambda-\lambda_{0}\right)^{2} \nu$. Thus if $\xi \in \mathscr{R}\left[\left(T-\lambda_{0} I\right)^{2}\right]$, then $\left(\lambda-\lambda_{0}\right)^{-2} \in L_{1}(\mu)$.

Conversely if $\left(\lambda-\lambda_{0}\right)^{-2} \in L_{1}(\mu)$, writing $\eta=\int_{C}\left(\lambda-\lambda_{0}\right)^{-2} \mu(d \lambda)$ we have

$$
\begin{aligned}
\left(T-\lambda_{0} I\right)^{2} \eta & =\int_{C}\left(\lambda^{\prime}-\lambda_{0}\right)^{2} E\left(d \lambda^{\prime}\right) \eta \\
& =\int_{\lambda^{\prime} \in C} \int_{\lambda \in C}\left(\lambda^{\prime}-\lambda_{0}\right)^{2} E\left(d \lambda^{\prime}\right)\left(\lambda-\lambda_{0}\right)^{-2} E(d \lambda) \xi \\
& =\int_{C} E(d \lambda) \xi \\
& =\xi .
\end{aligned}
$$

(ii) $\left(\lambda-\lambda_{0}\right)^{-2} \in L_{1}(\mu)$ for all $\lambda_{0} \in G$ if and only if $\mu(G)=0$.

The "if" is obvious. For the "only if" let $C_{0} \subset C$ be a square, with sides of length $D$ parallel to the real and imaginary axes, together with its interior. Let $\left\{C_{n}\right\}$ be a sequence of similar squares defined inductively so that $C_{n+1}$ is one of the quarters of $C_{n}$ with $\left\|\mu\left(C_{n+1}\right)\right\| \geqq\left(\frac{1}{4}\right)\left\|\mu\left(C_{n}\right)\right\|$. Then

$$
\left\|\mu\left(C_{n}\right)\right\| \geqq 4^{-n}\left\|\mu\left(C_{0}\right)\right\| \geqq 0
$$

and the diameter of $C_{n}$ is $2^{-n} D \sqrt{ } 2$. Let $\lambda_{0}$ be the point common to the squares $C_{n}$. Then $\int_{C_{n}}\left|\lambda-\lambda_{0}\right|^{-2} d \mu \rightarrow 0$ as $n \rightarrow \infty$. However

$$
\begin{aligned}
\left\|\int_{C_{n}}\left|\lambda-\lambda_{0}\right|^{-2} d \mu\right\| & \geqq\left(2^{-n} D \sqrt{ } 2\right)^{-2}\left\|\mu\left(C_{n}\right)\right\| \\
& \geqq\left(2^{-n} D \sqrt{ } 2\right)^{-2} 4^{-n}\left\|\mu\left(C_{0}\right)\right\| \\
& =\left(2 D^{2}\right)^{-1}\left\|\mu\left(C_{0}\right)\right\| \\
& \geqq 0
\end{aligned}
$$

so that $\mu\left(C_{0}\right)=0$. Since $G$ can be written as the union of a sequence of such squares (e.g., those with rational vertices), we have $\mu(G)=0$.

(iii) $\mu(G)=0$ if and only if $\xi \in \mathcal{N}(E(G))$.

This follows directly from $\mu(G)=E(G) \xi$.

(i), (ii), and (iii) together give a proof of the theorem.

3.3. Definition. $\lambda$ is said to be a critical eigenvalue of $\left\{T_{1}, T_{2}\right\}$ if $\lambda \in \sigma_{p}\left(T_{2}\right)$ and $\mathscr{R}\left(T_{1}-\lambda I_{1}\right)$ is of infinite codimension.

Since $T_{1}$ is normal this is the same as saying that $\lambda \in \sigma\left(T_{1}\right) \cap \sigma_{p}\left(T_{2}\right)$ and $\lambda$ is not 
an isolated point of $\sigma\left(T_{1}\right)$ corresponding to a finite dimensional snace of eigenvectors.

3.4. ThEOREM. $S=B+K_{1}+\cdots+K_{n}$ where $B \in \mathscr{B}\left(H_{1}, H_{2}\right), \lambda_{1}, \ldots$, $\lambda_{n}$ are critical eigenvalues of $\left\{T_{1}, T_{2}\right\}$ and for each $i, K_{i}$ is a discontinuous map from $H_{1}$ into $\mathscr{N}\left(T_{2}-\lambda_{i} I_{2}\right)$ which is zero on $\mathscr{R}\left(T_{1}-\lambda_{i} I_{1}\right)$. The operators $K_{i}$ and $B$ all commute with $\left\{T_{1}, T_{2}\right\}$.

Proof. Call a point $\lambda \in C$ such that $E_{2}(N) S$ is discontinuous for every neighborhood $N$ of $\lambda$ a discontinuity value. A discontinuity value clearly lies in $\sigma\left(T_{2}\right)$. We first show that the set of discontinuity values is finite.

Suppose the discontinuity values form an infinite set. Then we can find a sequence $\lambda_{1}, \lambda_{2}, \ldots$ of discontinuity values and a neighborhood $N_{i}$ of each $\lambda_{1}$ such that the $N_{i}$ are disjoint. For each $i$, let $\xi_{i} \in H_{1}$ with $\left\|\xi_{i}\right\|<2^{-i}$ and $\left\|E_{2}\left(N_{i}\right) S \xi_{i}\right\|>2^{i}$. Put $\eta=\sum_{i=0}^{\infty} E_{1}\left(N_{i}\right) \xi_{i}$. By $2.4,2.5$ and 3.2 , for any open set $N$ in $C$,

$$
S \mathscr{R}\left(I_{1}-E_{1}(N)\right) \subset \mathscr{N}\left(E_{2}(N)\right),
$$

so that $E_{2}(N) S=E_{2}(N) S E_{1}(N)$. Hence

$$
\begin{aligned}
E_{2}\left(N_{i}\right) S \eta & =E_{2}\left(N_{i}\right) S E_{1}\left(N_{i}\right) \eta \\
& =E_{2}\left(N_{i}\right) S E_{1}\left(N_{i}\right) \xi_{i}=E_{2}\left(N_{i}\right) S \xi_{i}
\end{aligned}
$$

so that $\|S \eta\| \geqq\left\|E_{2}\left(N_{i}\right) S \eta\right\| \geqq 2^{i}$ for all $i$.

Put

$$
\mathscr{G}=\left\{G ; G \text { an open set in } C, E_{2}(G) S \text { is continuous }\right\} \text {. }
$$

If $\lambda_{1}, \lambda_{2}, \ldots, \lambda_{n}$ are the discontinuity values, $\bigcup \mathscr{G}=C \backslash\left\{\lambda_{1}, \ldots, \lambda_{n}\right\}$ and, since $\bigcup \mathscr{G}$ can be written as the union of a countable subset of $\mathscr{G}, I_{2}-E_{2}\left(\left\{\lambda_{1}, \ldots, \lambda_{n}\right\}\right)$ is the orthogonal projection whose null space is $\bigcap \mathscr{N}(E(G))(G \in \mathscr{G})$. Thus by 3.1 $B=\left[I_{2}-E_{2}\left(\left\{\lambda_{1}, \ldots, \lambda_{n}\right\}\right)\right] S$ is continuous.

Put $K_{i}=E_{2}\left(\left\{\lambda_{i}\right\}\right) S$. We then have $S=B+K_{1}+\cdots+K_{n}$. Also

$$
\begin{aligned}
B T_{1} & =\left[I_{2}-E_{2}\left(\left\{\lambda_{1}, \ldots, \lambda_{n}\right\}\right)\right] S T_{1} \\
& =\left[I_{2}-E_{2}\left(\left\{\lambda_{1}, \ldots, \lambda_{n}\right\}\right)\right] T_{2} S \\
& =T_{2}\left[I_{2}-E_{2}\left(\left\{\lambda_{1}, \ldots, \lambda_{n}\right\}\right)\right] S \\
& =T_{2} B .
\end{aligned}
$$

Similar calculations show that the $K_{i}$ commute with $\left\{T_{1}, T_{2}\right\}$. If $N_{i}$ is an open neighborhood of $\lambda_{i}$ not containing any other discontinuity values, then $E_{2}\left(N_{i}\right) S$ $=E_{2}\left(N_{i}\right) B+K_{i}$ is not continuous so that $K_{i}$ is not continuous. We have $\mathscr{R}\left(K_{i}\right)$ $\subset \mathscr{R}\left(E_{2}\left(\lambda_{i}\right)\right)=\mathscr{N}\left(T_{2}-\lambda_{i} I_{2}\right)$ and

$$
S \mathscr{R}\left(T_{1}-\lambda_{\imath} I_{1}\right) \subset \mathscr{R}\left(T_{2}-\lambda_{i} I_{2}\right) \subset \mathscr{N}\left(E_{2}\left(\lambda_{i}\right)\right)
$$

so that $K_{i}$ is zero on $\mathscr{R}\left(T_{1}-\lambda_{i} I_{1}\right)$.

Since $K_{i}$ is not continuous we have $\mathscr{R}\left(K_{i}\right) \neq 0$ and hence $\lambda_{i} \in \sigma_{p}\left(T_{2}\right)$. If $\mathscr{R}\left(T_{1}-\lambda_{i} I_{1}\right)$ were of finite codimension it would be closed (Lemma 2.3) and $K_{i}$ would be the 
composition of the natural quotient map $H_{1} \rightarrow H_{1} / \mathscr{R}\left(T_{1}-\lambda_{i} I_{1}\right)$ and a map from the finite dimensional space $H_{1} / \mathscr{R}\left(T_{1}-\lambda_{i} I_{1}\right)$ into $H_{2}$. Since both these maps are continuous $K_{i}$ would be continuous. Thus we have $\lambda_{i} \in \sigma_{p}\left(T_{2}\right)$ and $\mathscr{R}\left(T_{1}-\lambda_{i} I_{1}\right)$ of infinite codimension, that is $\lambda_{i}$ is a critical eigenvalue.

3.5. Corollary. $S$ can be discontinuous if and only if $\left\{T_{1}, T_{2}\right\}$ has critical eigenvalues.

Proof. Follows from 2.1, 2.2, 3.3 and 3.4.

3.6. Corollary. If $S T=T S$ then $S$ can be discontinuous if and only if either $\sigma_{p}(T)$ contains points corresponding to eigenspaces of infinite dimension or $\sigma_{p}(T)$ contains points which are not isolated points of $\sigma(T)$.

4. Operators commuting with sets of normal operators. Suppose now that $S$ satisfies a relationship $S T_{1}=T_{2} S$ for a number of different pairs of normal operators. Theorem 3.4 then gives a decomposition of $S$ for each pair and the question of when this implies the continuity of $S$ arises. It would be pleasant to be able to deduce that, for $S$ to be discontinuous, the pairs with which it commutes must have critical eigenvectors in common. The following example shows that this need not be so.

Let $e_{1}, e_{2}, \ldots$ be a complete orthonormal sequence in the separable Hilbert space $H$ and $C$ the compact operator determined by $C e_{1}=0, C e_{n}=n^{-1} e_{n}, n=2$, $3, \ldots .0$ is the only critical eigenvalue of $\{C, C\}$, the corresponding eigenvector being $e_{1}$. Let $S$ be a discontinuous linear map of $H$ into $H$ with $S C=C S$. The unitary operator $U ; H \oplus H \rightarrow H \oplus H$ defined by $U(\xi, \eta)=(\eta, \xi)$ has critical eigenvalues \pm 1 with corresponding spaces $\{(\xi, \xi) ; \xi \in H\}$ and $\{(\xi,-\xi) ; \xi \in H\}$. We have

$$
\begin{gathered}
U\left[\begin{array}{l}
S, 0 \\
0, S
\end{array}\right]=\left[\begin{array}{l}
0, S \\
S, 0
\end{array}\right]=\left[\begin{array}{l}
S, 0 \\
0, S
\end{array}\right] U \text { and }\left[\begin{array}{l}
C, 0 \\
0, I+C
\end{array}\right]\left[\begin{array}{l}
S, 0 \\
0, S
\end{array}\right]=\left[\begin{array}{l}
S, 0 \\
0, S
\end{array}\right]\left[\begin{array}{l}
C, 0 \\
0, I+C
\end{array}\right], \\
{\left[\begin{array}{l}
S, 0 \\
0, S
\end{array}\right] \text { discontinuous but } U \text { and }\left[\begin{array}{l}
C, 0 \\
0, I+C
\end{array}\right]}
\end{gathered}
$$

have no common eigenvectors.

In particular the decomposition of $S$ given by Theorem 3.4 is not unique. Applying 3.1 to $S\left(S, T_{1}, T_{2}\right.$ as at the beginning of $\left.\S 3\right)$ with $\left\{P_{\alpha} ; \alpha \in A\right\}$ as the set of all orthogonal projections $P$ in $H_{2}$ with $P S$ continuous we obtain the space $N$ which depends on $S$ only. Theorem 3.4 then shows that $N$ is in $\mathscr{R}\left(E_{2}\left(\left\{\lambda_{1}, \lambda_{2}, \ldots, \lambda_{n}\right\}\right)\right)$ a space spanned by the eigenvectors of $T_{2}$ corresponding to a finite number of critical eigenvalues of $\left\{T_{1}, T_{2}\right\}$. We can prove rather more.

4.1. THEOREM. $N$ is spanned by the eigenvectors of $T_{2}$ which it contains.

Proof. $N^{\perp}=\{x ; y \rightarrow(S y, x)$ is continuous $\}$.

Let $x=x_{1}+\cdots+x_{n} \in N^{\perp}$ where, for each $i, x_{i} \in \mathscr{N}\left(T_{2}-\lambda_{i} I_{2}\right)$. (The $\lambda_{i}$ are, as in 3.4 , the discontinuity values.) We shall prove that each $x_{i} \in N^{\perp}$. Suppose $x_{1} \notin N^{\perp}$ 
and thus that $f: y \rightarrow\left(S y, x_{1}\right)$ is not continuous. Let $V$ be an open neighborhood of $\lambda_{1}$ with $\lambda_{i} \notin \bar{V}^{-1}, i=2,3, \ldots, n$. We have

and

$$
\begin{aligned}
S \mathscr{R}\left(T_{1}-\lambda_{i} I_{1}\right) \subset \mathscr{R}\left[\left(T_{2}-\lambda_{i} I_{2}\right) S\right] & \subset \mathscr{N}\left(E_{2}\left(\left\{\lambda_{1}\right\}\right)\right) \\
& \subset\left\{x_{i}\right\}^{\perp}, \quad i=1, \ldots, n,
\end{aligned}
$$

$$
\mathscr{N}\left(E_{1}(V)\right) \subset \mathscr{R}\left(T_{1}-\lambda_{1} I_{1}\right)
$$

so that $f$ is zero on $\mathscr{N}\left(E_{1}(V)\right)$ and thus discontinuous on $\mathscr{R}\left(E_{1}(V)\right)$. However we also have

$$
\mathscr{R}\left(E_{1}(V)\right) \subset \mathscr{R}\left(T_{1}-\lambda_{l} I_{1}\right), \quad i=2, \ldots, n,
$$

so that $y \rightarrow\left(S y, x_{i}\right)$ is zero on $\mathscr{R}\left(E_{1}(V)\right)$ for $i=2, \ldots, n$, and so, on that space, $f(y)=(S y, x)$, which is impossible as $f$ is not continuous there.

Hence

$$
N^{\perp}=\mathscr{R}\left(I_{2}-E_{2}\left(\left\{\lambda_{1}, \ldots, \lambda_{n}\right\}\right)\right)+\sum_{i=1}^{n}\left[\mathscr{N}\left(T_{2}-\lambda_{l} I_{2}\right) \cap N^{\perp}\right]
$$

so that, since the spaces $\mathscr{N}\left(T_{2}-\lambda_{i} I_{2}\right), i=1, \ldots, n$, and $\mathscr{R}\left(I_{2}-E_{2}\left(\left\{\lambda_{1}, \ldots, \lambda_{n}\right\}\right)\right)$ are mutually orthogonal,

$$
N=N^{\perp \perp}=\sum_{i=1}^{n}\left[\mathcal{N}\left(T_{2}-\lambda_{i} I_{2}\right) \cap N\right] .
$$

A necessary condition therefore for there to exist a discontinuous linear map $S$ commuting with every element of a set $\left\{\left\{T_{1, \alpha}, T_{2, \alpha}\right\} ; \alpha \in A\right\}$ of pairs of normal operators is that there should exist a subspace $N$ of $H_{2}$ of nonzero dimension which, for each $\alpha$, is spanned by eigenvectors of $T_{2, \alpha}$ corresponding to a finite number of critical eigenvalues of $\left\{T_{1, \alpha}, T_{2, \alpha}\right\}$. This condition is not sufficient.

5. Operators commuting with scalar type operators. We refer the reader to $[3, p$. 226] for a definition of scalar type operator. Let now $H_{1}, H_{2}$ be Banach spaces, $T_{i} \in \mathscr{B}\left(H_{i}\right)$, a scalar type operator for $i=1,2$ and $S$ a linear map from $H_{1}$ into $H_{2}$ with $S T_{1}=T_{2} S$. Theorem 3.4 applies to this situation but the proofs of the results on which it is based require slight changes. Lemma 3.1 is replaced by

5.1. Lemma. Let $A$ be an index set, $L$ a linear map $H_{1} \rightarrow H_{2}$ and, for each $\alpha \in A$, let $P_{\alpha}$ be a bounded projection in $H_{2}$ with $P_{\alpha} L$ continuous. Let $N=\bigcap \mathscr{N}\left(P_{\alpha}\right)(\alpha \in A)$ and $P$ a bounded projection in $H_{2}$ with $\mathscr{N}(P) \subset N$. Then $P L$ is continuous.

Theorem 3.2 applies to the present case. In the proof of (ii) it is easier to consider the scalar measures $\nu(X)=f(\mu(X))=f(E(X) \xi)$ where $f \in H_{1}^{*}$. The proof then shows that $\left(\lambda-\lambda_{0}\right)^{-2} \in L_{1}(v)$ for all $\lambda_{0} \in G$ if and only if $f(\mu(G))=0$. Since however we obtain this for all $f \in H_{1}^{*}$ the result (ii) follows. The proof of Theorem 3.4 now goes through with little change.

6. Operators commuting with restrictions of normal operators. If $H_{1}, H_{2}$ are again Hilbert spaces, $T_{1}, T_{2}$ normal operators in $H_{1}, H_{2}$, which need not be bounded (see [4, p. 1258]) and $S$ is a linear map from $H_{1}$ into $H_{2}$ with $S T_{1} \subseteq T_{2} S$ then $S \mathscr{D}\left(T_{1}\right) \subset \mathscr{D}\left(T_{2}\right)$ and the results and proofs in $\S 3$ apply without change.

Sometimes it is possible to apply Theorem 3.4 to a situation in which $T_{1}, T_{2}$ are not normal operators by extending them to a larger space in which they are. It is 
easy to see that if $T_{2}$ is an operator which can be extended to a normal operator $T_{3}$ in a larger space $H_{3}$ then $S T_{1}=T_{3} S$ and 3.4 can be applied. Cases involving the extension of $T_{1}$ are rather more difficult since in these $S$ has to be extended. We give an example of a case in which this extension is possible.

Let now $H_{1}$ be the subspace of $H_{0}=L_{2}\left(\left(1+t^{2}\right) \lambda\right)$, where $\lambda$ is Lebesgue measure on $(-\infty,+\infty)$, consisting of boundary values of functions analytic in the upper half plane (this space is similar to Hoffman's $\tilde{H}^{2}$ in [5, p. 131]). For $i=0,1$, let $T_{i}$ be the operator in $H_{i}$ defined by $\left(T_{i} f\right)(t)=t f(t), \mathscr{D}\left(T_{i}\right)$ being the set of all $f$ in $H_{i}$ for which $t f(t) \in H_{i}$. Let $\phi_{n}(t)=\pi^{-1 / 2}(t-i)^{n}(t+i)^{-n}, n$ an integer. Then $\phi_{n}, n=0,1, \ldots$, is an orthonormal basis for $H_{1}$ (the transformation $f(t) \rightarrow f\left(i(1+z)(1-z)^{-1}\right.$ ) is a unitary transformation of $H_{1}$ onto $H^{2}$ and of $H_{0}$ onto $L^{2}$ of the circle), and the shift operator $U \phi_{n}=\phi_{n+1}$ is the Cayley transform of $T_{1} . T_{1}$ is in fact an elementary symmetric operator $\left(\left[4\right.\right.$, p. 1272]) and $T_{0}$ a self-adjoint operator.

Let now $H_{2}$ be a Hilbert space, $T_{2}$ a self-adjoint operator on $H_{2}$ and $S$ a linear map of $H_{1}$ into $H_{2}$ with $S T_{1} \subseteq T_{2} S$. Put $f^{*}(t)=[f(\bar{t})]^{-}$. The subspaces $H_{1}, H_{1}^{*}$ of $H_{0}$ then have only the constant function in common and together span $H_{0}$. If $f \in H_{0}$ we write $f=f_{+}+f_{-}$where $f_{+} \in H_{1}, f_{-} \in H_{1}^{*}$, are indeterminate up to a constant. Since

if and only if

$$
\sum_{i=0}^{\infty} \xi_{i} \phi_{i} \in \mathscr{D}\left(T_{1}\right)
$$

and

$$
\sum_{j=0}^{\infty}\left|\sum_{i=j}^{\infty} \xi_{i}\right|^{2}<\infty
$$

if and only if

$$
\sum_{i=-\infty}^{+\infty} \xi_{i} \phi_{i} \in \mathscr{D}\left(T_{0}\right)
$$

$$
\sum_{j=-\infty}^{\infty}\left|\sum_{i=j}^{\infty} \xi_{i}\right|^{2}<\infty,
$$

for $f \in \mathscr{D}\left(T_{0}\right)$ there is exactly one choice of $f_{+}, f_{-}$with $f_{+}, f_{-}^{*} \in \mathscr{D}\left(T_{1}\right)$.

Take a conjugation \# on $H_{2}$ commuting with $T_{2}$ for which $\left(S \phi_{0}\right)^{\#}=S \phi_{0}$ (such a conjugation can be constructed by taking a spectral representation $U$ of $T_{2}$ as in [4, p. 1205], in which $S \phi_{0}$ is a real valued function and then writing $x^{\#}=U^{-1}(U x)^{-}$ where for $\left.\xi \in \sum L^{2}\left(\mu_{a}\right), a \in A, \xi_{a}(t)=\left(\xi_{a}(t)\right)^{-}\right)$. Now put $S_{0} f=S f_{+}+\left(S f_{-}^{*}\right)^{\#} . S_{0}$ is a linear map from $H_{0}$ into $H_{2}$ and is well defined since $\left(S \phi_{0}^{*}\right)^{\#}=S \phi_{0}$.

Let $f \in \mathscr{D}\left(T_{0}\right)$. Then

$$
\begin{aligned}
S_{0} T_{0} f & =S_{0} T_{0}\left(f_{+}+f_{-}\right) \\
& =S_{0} T_{1} f_{+}+S_{0}\left(T_{1} f_{-}^{*}\right)^{*} \\
& =S T_{1} f_{+}+\left(S T_{1} f_{-}^{*}\right)^{\#} \\
& =T_{2} S f_{+}+T_{2}\left(S f_{-}^{*}\right)^{\#} \\
& =T_{2} S_{0} f .
\end{aligned}
$$


We thus have $S_{0} T_{0} \subseteq T_{2} S_{0}$. Since the spectrum of $S_{0}$ is the whole real line the extended version of 3.4 discussed at the beginning of this section applies to show that if $\sigma_{p}\left(T_{2}\right)$ is void $S_{0}$ and hence $S$ must be continuous. If $\sigma_{p}\left(T_{2}\right)$ is not void $S$ can be discontinuous.

The case $S T_{1} \subseteq T_{2} S$ with $T_{2}$ self-adjoint and $T_{1}$ a maximal symmetric operator with deficiency indices $d_{+}=0, d_{-}=n, n$ finice, can be shown to lead to the same result by decomposing $H_{1}$ into a direct sum $\mathfrak{S}_{0} \oplus \mathfrak{S}_{1} \oplus \cdots \oplus \mathfrak{S}_{n}$ where $T_{1}$ is selfadjoint on $\mathfrak{S}_{0}$ and unitarily equivalent to the elementary symmetric operator considered above on $\mathfrak{S}_{1}, \ldots, \mathfrak{S}_{n}$. The case $d_{+}=n, d_{-}=0$ is solved by transferring to $-T_{1}$ whose indices are $d_{+}=0, d_{-}=n$ (see [4, p. 1272]).

These methods can also be applied to isometric operators to obtain similar results. In particular we can show that the isometric shift operator in $l_{2}(0, \infty)$ has the property that any map commuting with it is necessarily continuous-a result which can easily be seen directly and which also follows from Theorem 9.1. The author does not know whether the same applies to linear maps $S$ in $l_{2}(0, \infty)$ commuting with the adjoint of the shift operator (that is the operation of shifting one place to the left, the $a_{0}$ coordinate being dropped).

7. Operators commuting with centralisers. In the following we use the terms regular and hermitian involution as defined in [11, p. 219] and [12, p. 189], respectively.

7.1. THEOREM. Let $A$ be a regular commutative semisimple Banach algebra with identity and hermitian involution. Let $x \in A, J$ a closed ideal in $A$ and $G$ an open set in C. Then

$$
\bigcap_{\lambda \in G}(x-\lambda e) J=k^{\prime}\left(\hat{x}^{-1}(G)\right)
$$

(For any set $X$ in $\mathscr{M}_{A}$, the maximal ideal space of $A$,

$$
\left.k^{\prime}(X)=\{j ; j \in J, \hat{j}(M)=0 \text { for all } M \in X\}\right) .
$$

Proof. The second set clearly contains the first. Suppose $\lambda_{0} \in G$ and $j \in k^{\prime}\left(\hat{x}^{-1}(G)\right)$. We shall show that the function $\hat{a}(M)=\hat{j}(M) /\left(\hat{x}(M)-\lambda_{0}\right)$, taken as 0 when $\hat{x}(M)=\lambda_{0}$, is in fact the transform of some element $a$ of $J$. Since $G$ is open and $\hat{x}$ continuous, $\hat{a}(M)=0$ for $M \in \hat{x}^{-1}(G)$, and so $\hat{a}$ belongs to $J$ locally at all points of $\hat{x}^{-1}(G)$. Now suppose $\hat{x}\left(M_{0}\right) \neq \lambda_{0}$. Again using the properties of $G$ and $\hat{x}$ we can find a neighborhood $N$ of $M_{0}$ with $\left|\hat{x}\left(M_{0}\right)-\hat{x}(M)\right| \leqq\left|\hat{x}\left(M_{0}\right)-\lambda_{0}\right|$, and a closed neighborhood $V$ of $M_{0}$ with $V \subset N$. Using the corollary on p. 224 of [11] we take $c \in A$ such that $\hat{c}(M)=1$ for $M \in V$ and $\hat{c}(M)=0$ for $M \notin N$. Replacing $c$ by $c c^{*}$ if necessary we can assume that $\hat{c}$ is nonnegative real valued. Let now

$$
\alpha=\exp \left\{-i \operatorname{Arg}\left(\hat{x}\left(M_{0}\right)-\lambda_{0}\right)\right\} \text {. }
$$

The function $(1-\hat{c})+i \alpha\left(\hat{x}-\lambda_{0}\right) \hat{c}$ takes the value 1 outside $N$ whereas on $N$,

$$
\left|\alpha\left(\hat{x}(M)-\lambda_{0}\right)-\alpha\left(\hat{x}\left(M_{0}\right)-\lambda_{0}\right)\right|<\alpha\left(\hat{x}\left(M_{0}\right)-\lambda_{0}\right),
$$

so that $\alpha\left(\hat{x}(M)-\lambda_{0}\right)$ has positive real part and thus $(1-\hat{c})+i \alpha\left(\hat{x}-\lambda_{0}\right) \hat{c}$ is not zero on $N$ either. Using Wiener's theorem we see that $-i \alpha^{-1}(e-c)+\left(x-\lambda_{0} e\right) c$ is 
regular and, writing $b$ for its inverse, $(j b)^{\wedge}=\hat{a}$ on $V$. This shows that $\hat{a}$ belongs to $J$ locally at all points of $\mathscr{M}_{A}$ and so, by Theorem $3^{\prime}$ on p. 225 of [11] there exists $a \in J$ whose Gel'fand transform is $\hat{a}$. Clearly $\left(x-\lambda_{0} e\right) a=j$.

7.2. RemarK. The hermitian involution was used only to show that $c$ could be taken to be real and so to construct a nowhere zero function in $A^{\wedge}$ equal to $\hat{x}-\lambda_{0}$ in $V$. The same end can be achieved by supposing instead that the functions $c$ are bounded (by $K$ say) independently of $N$ and $V$. In this case we choose $N$ such that

$$
\left|\hat{x}(M)-\hat{x}\left(M_{0}\right)\right|<K^{-1}\left|\hat{x}\left(M_{0}\right)-\lambda_{0}\right| \text { for all } M \in N
$$

and then we see that

$$
\left(\hat{x}\left(M_{0}\right)-\lambda_{0}\right)(1-\hat{c}(M))+\left(\hat{x}(M)-\lambda_{0}\right) \hat{c}=\hat{x}\left(M_{0}\right)-\lambda_{0}+\hat{c}(M)\left(\hat{x}(M)-\hat{x}\left(M_{0}\right)\right)
$$

has the required properties.

We now state a result which is an extension of Lemma 3.1, and is proved by the same method.

7.3. Lemma. Let $E, F$ be two Banach spaces, $S$ a linear map from $E$ to $F$ and $I$ an index set. For each $\iota \in I$ let $E_{\imath}$ be a Banach space and $P_{\imath}$ a continuous linear map from $F$ into $E_{\iota}$ such that $P_{\iota} S$ is continuous. Put $N=\bigcap \mathcal{N}\left(P_{\iota}\right)(\iota \in I)$ and let $P$ be the canonical map of $F$ onto $F / N$. Then PS is continuous.

If $x$ is an element of an algebra $A$ we shall write $L_{x}$ for the operator $L_{x}(a)=x a$ for all $a \in A$.

7.4. THEOREM. Let $A, B$ be two commutative Banach algebras with identity, $x, y$ elements and $J, K$ ideals in $A, B$ respectively. Suppose $A$ is regular and has a hermitian involution and that $B$ is semisimple. Let $S$ be a linear map $J \rightarrow K$ commuting with $\left\{L_{x}, L_{y}\right\}$. If $\left\{L_{x}, L_{y}\right\}$ has no critical eigenvalue then $S$ is continuous.

Proof. The proof is similar to that of 3.4. For any set $X$ in $C, E_{2}(X)$ is defined as the canonical map of $B$ into $B / k^{\prime}\left(\hat{y}^{-1}(X)\right)$. The definition of discontinuity value is then as before.

In proving that the set of discontinuity values is finite we choose the open sets $N_{i}$ in $C$ such that $N_{i}$ and $\bigcup_{(j \neq i)} N_{j}$ have disjoint closures, and then take $c_{i} \in A$ with $\hat{c}_{i}=1$ in $\hat{x}^{-1}\left(N_{i}\right)$ and $\hat{c}_{i}=0$ in $\hat{x}^{-1}\left[\bigcup_{(j \neq i)} N_{j}\right]$. Now take $\xi_{j} \in J$ with $\left\|\xi_{j}\right\|<2^{-j}\left\|c_{j}\right\|^{-1}$ and $\left\|E_{2}\left(N_{j}\right) S \xi_{j}\right\|>2^{j}$, and put $\eta=\sum c_{i} \xi_{i}$. Using 2.5 and 7.1 we see that

$$
S k^{\prime}\left(\hat{x}^{-1}(G)\right) \subset k^{\prime}\left(\hat{y}^{-1}(G)\right)
$$

for any open set $G$ in $C$. Applying this to $\eta-c_{i} \xi_{i} \in k^{\prime}\left(\hat{x}^{-1}\left(N_{i}\right)\right)$, we have $E_{2}\left(N_{i}\right) S \eta$ $=E_{2}\left(N_{i}\right) S c_{i} \xi_{i}$. However $\left(e-c_{i}\right) \xi_{i} \in k^{\prime}\left(\hat{x}^{-1}\left(N_{i}\right)\right)$ so that

$$
E_{2}\left(N_{i}\right) S c_{i} \xi_{i}=E_{2}\left(N_{i}\right) S \xi_{i} .
$$

These together imply $\left\|E_{2}\left(N_{i}\right) S \eta\right\|>2^{i}$ for all $i$, which is impossible since the $E_{2}\left(N_{i}\right)$ are all of norm 1 . Thus there are only finitely many discontinuity values $\lambda_{1}, \ldots, \lambda_{n}$. 
Write $\mathfrak{X}=\left\{X ; X \subset C, E_{2}(X) S\right.$ is continuous $\}$. Lemma 7.3 shows that $E_{2}(\bigcup \mathfrak{X}) S$ is continuous. We have shown that $\bigcup \mathfrak{X} \supset \boldsymbol{C} /\left\{\lambda_{1}, \ldots, \lambda_{n}\right\}$ so that $\cup \mathfrak{X}=\boldsymbol{C} \backslash\left\{\lambda_{1}, \ldots, \lambda_{n}\right\}$ and $E_{2}\left(\left\{\lambda_{i}\right\}\right) S$ is discontinuous for each $\lambda_{i}$. Since

$$
k^{\prime}\left(\mathscr{M}_{B} \mid \hat{y}^{-1}\left\{\lambda_{1}, \ldots, \lambda_{n}\right\}\right)=k^{\prime}\left(\mathscr{M}_{B} \mid \text { int } \hat{y}^{-1}\left\{\lambda_{1}, \ldots, \lambda_{n}\right\}\right),
$$

this implies that, for each $i, \hat{y}^{-1}\left(\left\{\lambda_{i}\right\}\right)$ has interior points and so $\lambda_{i} \in \sigma_{p}\left(L_{y}\right)$. Now if $\lambda \in \rho\left(L_{x}\right)$ and $N$ is an open neighborhood of $\lambda$ in $\rho\left(L_{x}\right)$ then $k^{\prime}\left(\hat{x}^{-1}(N)\right)=J$ and $E_{2}(N) S=0$, which implies that $\lambda_{i} \in \sigma\left(L_{x}\right)$. However $\left\{L_{x}, L_{y}\right\}$ has no critical eigenvalues so that for each $i, \hat{x}^{-1}\left(\left\{\lambda_{i}\right\}\right)$ contains a finite number of isolated points of $\mathscr{M}_{A}$. If now $c$ is the characteristic function of $\hat{x}^{-1}\left(\left\{\lambda_{i}\right\}\right)$ we have

$$
c j \in k^{\prime}\left(\hat{x}^{-1}\left(C \backslash\left\{\lambda_{i}\right\}\right)\right) \text { and }(e-c) j \in k^{\prime}\left(\hat{x}^{-1}\left(\left\{\lambda_{i}\right\}\right)\right)
$$

for all $j \in J$. This shows that $E_{2}\left(\left\{\lambda_{i}\right\}\right) S=E_{2}\left(\left\{\lambda_{i}\right\}\right) S L_{c}$ which is continuous since $L_{c}$ is continuous and has a finite dimensional range. This is a contradiction showing that there are no discontinuity values and $S=E_{2}(\bigcup \mathfrak{X}) S$ is continuous.

Suppose now that $A_{0}, B_{0}$ are two Banach algebras, $T_{1}, T_{2}$ centralisers on $A_{0}, B_{0}$ and $S$ a linear map $A_{0} \rightarrow B_{0}$ commuting with $\left\{T_{1}, T_{2}\right\}$. We wish to deduce from 7.4 that, if $\left\{T_{1}, T_{2}\right\}$ has no critical eigenvalues then $S$ must be continuous. The task is to construct suitable algebras $A, B$ containing $A_{0}, B_{0}$ as ideals and elements $x, y$ such that, on $A_{0}, B_{0}$, we have $T_{1}=L_{x}, T_{2}=L_{y}$. In order that $A, B$ satisfy the conditions of 7.4 it is necessary for $A_{0}$ to be regular and $B_{0}$ to be semisimple (see Theorem 2.7.2 and Corollary 2.3.7 of [12]). If this is so we can always take $B=\Gamma\left(B_{0}\right)$ (corollary on p. 305 of [6]). The choice for $A$ is more delicate. Of course if $T_{1}$ is actually multiplication by an element of $A_{0}$ then, providing $A_{0}$ has hermitian involution, we take $A$ as $A_{0}$, with identity adjoined if necessary. If $A_{0}$ is semisimple and has a hermitian involution this has a unique extension to $\Gamma\left(A_{0}\right)$ (Theorem 6 on p. 303 of [6]). If this involution is hermitian on $\Gamma\left(A_{0}\right)$ and $\Gamma\left(A_{0}\right)$ is regular then we take $A$ as $\Gamma\left(A_{0}\right)$. The celebrated result of Williamson [14] shows that $\Gamma\left(A_{0}\right)$ need not inherit these properties from $A_{0}$. The following result can sometimes be used.

7.5. Lemma. Let $T \in \Gamma\left(A_{0}\right)$ and $C$ be a subalgebra of $\Gamma\left(A_{0}\right)$ containing $T$. Let $A_{0}$ and $C$ be regular and let $A_{0}$ have a hermitian involution which induces a hermitian involution on $C$. Then the subalgebra $A$ of $\Gamma\left(A_{0}\right)$ containing the identity spanned by $A_{0} \cup C$ is regular and has a hermitian involution.

Proof. We suppose $C$ contains the identity of $\Gamma\left(A_{0}\right)$ since it can easily be added without altering the properties of $C$. If $\phi$ is a multiplicative linear functional on $A$ its restrictions to $A_{0}$ and $C$ are multiplicative linear functionals and so $\phi\left(x^{*}\right)=\phi(x)^{-}$ for all $x$ in $A_{0} \cup C$. Since $\phi$ is continuous this identity extends to $A$.

For the regularity we adapt the proof of Corollary 3.7.4 of [12]. Let $B$ denote $A_{0}$ with identity adjoined. $\hat{B} \cup \hat{C}$ strongly separates the points of $\mathscr{M}_{A}$ so that if $\phi_{0} \in \mathscr{M}_{A}$ and $F$ is a closed set in $\mathscr{M}_{A}$ not containing $\phi_{0}$ there are elements 
$b_{1}, \ldots, b_{m}$ of $B$ and $c_{1}, \ldots, c_{n}$ of $C$ and $\varepsilon>0$ such that $V_{\phi_{0}}\left(b_{1}, \ldots, b_{m}, c_{1}, \ldots, c_{n} ; \varepsilon\right)$ is disjoint from $F$. Define

$$
F_{B}=\left\{\psi ; \psi \in \mathscr{M}_{B},\left|\hat{b}_{i}(\psi)-\hat{b}_{\imath}\left(\phi_{0}\right)\right| \geqq \varepsilon \text { for some } i\right\}
$$

and similarly for $F_{C}$. By the regularity of $B$ there is $u_{B} \in B \subset A$ with $\hat{u}_{B}=0$ on $F_{B}$ and $\hat{u}_{B}\left(\phi_{0}\right) \neq 0$, and a corresponding element $u_{C}$ of $C$. The Gel'fand transform of the element $u=u_{B} u_{C}$ of $A$ is then not zero at $\phi_{0}$ but is zero outside $V_{\phi_{0}}$.

In using Lemma 7.5 it is sometimes possible to take $C=R\left(T, T^{*}\right)$, the subalgebra of $\Gamma\left(A_{0}\right)$ rationally generated by $\left\{T, T^{*}\right\}$ (see [12, p. 164]). For the involution on $\boldsymbol{R}\left(T, T^{*}\right)$ to be hermitian it is necessary and sufficient for $T^{* \wedge}=T^{\wedge}$ - .

These methods fail completely if $T$ is a centraliser with $T=T^{*}$ and yet $\sigma(T)$ is not real.

8. Operators commuting with translations. In this section we shall consider operators $S$ on spaces of functions on $(-\infty,+\infty)$ commuting with the translation operators $\delta_{a} ; f(x) \rightarrow f(x+a)$. In the space $L_{1}(-\infty,+\infty)$ the translation operators are centralisers in the algebra formed by taking convolution multiplication, this algebra being a regular semisimple Banach algebra with a hermitian involution. If $a \neq 0$ the subalgebra $C=\boldsymbol{R}\left(\delta_{a}\right)$ of $\Gamma\left(L_{1}\right)$ is * isomorphic with the group algebra of the integers, which is regular and has a hermitian involution. If $a \neq 0, \sigma_{p}\left(\delta_{a}\right)$ is void so that by Theorem 7.4 and Lemma 7.5 we have

8.1. Proposition. If $S$ is a linear map $L_{1}(-\infty,+\infty) \rightarrow L_{1}(-\infty,+\infty)$ such that $S \delta_{a}=\delta_{a} S$ for some $a \neq 0$ then $S$ is continuous.

Notes. (i) It is easy to see what $S$ must be like. If $S_{0}$ is any bounded linear function from $L_{1}(0,|a|)$ into $L_{1}(-\infty,+\infty)$ and $c$ the characteristic function of $(0,|a|)$ then $S=\sum_{i=-\infty}^{\infty} \delta_{i a} S_{0}\left[c \cdot \delta_{-i a} f\right]$ is a bounded operator in $L_{1}(-\infty,+\infty)$ commuting with $\delta_{a}$ and any operator commuting with $\delta_{a}$ must be of this form (with $S_{0}$ as the restriction of $S$ to $L_{1}(0,|a|)$ ).

(ii) If $S$ commutes with all the $\delta_{a}$ then it certainly is continuous. Using the fact that linear combinations of the $\delta_{a}$ are dense in $\Gamma\left(L_{1}\right)$ with the strong operator topology (see [7, p. 607]), we see that $S(\mu * f)=\mu * S f$ for all $f \in L_{1}$ and bounded measures $\mu$. In particular $S$ is a centraliser on $L_{1}$ and thus of the form $S f=\nu * f$ for some measure $\nu[13$, p. 252].

(iii) It would be interesting to know whether $S(\mu * f)=\mu * S f$ for all $f \in L_{1}$, where $\mu$ is a fixed bounded measure on $(-\infty,+\infty)$ whose Fourier-Steiltjes transform is not constant in any open interval, implies that $S$ is continuous.

(iv) In $8.1 L_{1}$ can be replaced by $L_{2}$ and the result follows from 3.4 since $\delta_{a}$ is a unitary operator. The proof of 7.4 can be adapted to give the same result in $L_{p}(-\infty,+\infty)$, the changes being relatively trivial for $1<p<2$ but requiring the introduction of distributions as Fourier transforms of some elements of $L_{p}$ for $p>2$. 
We now give an example which shows that in a Fréchet space the situation can be quite different. We consider the space $\mathscr{C}(-\infty,+\infty)$ with the topology of uniform convergence on finite intervals. For each $a \neq 0, \sigma_{p}\left(\delta_{a}\right)=C$ and for each $\alpha \neq 0$ the function $e^{\alpha x}$ is an eigenvector of all the $\delta_{a}$. The method of constructing a discontinuous commutant in $\S 2$ fails however as $\mathscr{R}\left(\delta_{a}-\lambda I\right)=\mathscr{C}$ for all $a, \lambda$ except $a=0, \lambda=1$.

We shall show that there is a discontinuous linear transformation on $\mathscr{C}$ which commutes with all the $\delta_{a}$ (it is easy to find one which commutes with a given $\delta_{a}$ ). The subspace $\mathscr{C}_{e}$ of $\mathscr{C}$ consisting of functions $f$ with $|f(x)|=O\left(e^{|k x|}\right)$ as $x \rightarrow \pm \infty$ for some $k$ is closed with respect to the operators $\delta_{a}$. Furthermore the equation $\sum \lambda_{i} \delta_{a_{1}} f=g(*)$, where $\lambda_{1}, \ldots, \lambda_{n} \in C, a_{1}, a_{2}, \ldots, a_{n} \in(-\infty,+\infty)$ and $g \in \mathscr{C}_{e}$ always has a solution $f \in \mathscr{C}_{e}$. It follows from this that linear combinations of the $\delta_{a}$ together with their inverses form a field $K$ acting on $\mathscr{C} \mid \mathscr{C}_{e}$, that is $\mathscr{C} \mid \mathscr{C}_{e}$ is a vector space over $K$. The equation $*$ has a unique solution $f \in \mathscr{C}_{+}$whenever $g \in \mathscr{C}_{+}$, the subspace of $\mathscr{C}$ consisting of functions whose support is limited to the left. Thus $\mathscr{C}_{+}$ becomes a vector space over $K$. If now $S_{0}$ is a nonzero $K$-linear transformation from $\mathscr{C} / \mathscr{C}_{e}$ to $\mathscr{C}_{+}$and we define $S=S_{0} Q$ where $Q$ is the canonical map $\mathscr{C} \rightarrow \mathscr{C} \mid \mathscr{C}_{e}$ then $S$ commutes with all the $\delta_{a}$ and is discontinuous since it is not zero but is zero on the dense subset $\mathscr{C}_{e}$ of $\mathscr{C}$.

9. Operators whose ranges are sequence spaces. In this section $B K$ space is as defined in $[15$, p. 29]. An operator in such a space given by a matrix is continuous $[15$, p. 29]. We now prove a theorem concerning operators commuting with continuous operators which is totally different from the previous results since it does not depend on dividing the domain space by dividing the spectrum of $T_{1}$, indeed the operator $T_{1}$ can be chosen to be nilpotent.

9.1. Theorem. Let $E_{1}$ be a Banach space, $E_{2}$ a BK space. Let $T_{1}$ be a continuous linear operator in $E_{1}$ and $T_{2}$ an operator in $E_{2}$ generated by a triangular matrix $\left[a_{i j}\right]$. Suppose that $\sigma_{p}\left(T_{2}\right)$ is void. Let $S$ be a linear map from $E_{1}$ into $E_{2}$ with $S T_{1}=T_{2} S$. Then $S$ is continuous.

Proof. For $\xi \in E_{1}$

$$
\left(S T_{1} \xi\right)_{n}=\left(T_{2} S \xi\right)_{n}=a_{n, 1}(S \xi)_{1}+a_{n, 2}(S \xi)_{2}+\cdots+a_{n, n}(S \xi)_{n}
$$

So that

$$
\left(S\left(T_{1}-a_{n, n} I_{1}\right) \xi\right)_{n}=a_{n, 1}(S \xi)_{1}+\cdots+a_{n, n-1}(S \xi)_{n-1} .
$$

From this we can show by induction on $n$ that $\left(S P_{n} \xi\right)_{m}=0$ for $m \leqq n$ where

$$
P_{n}=\left(T_{1}-a_{1,1} I_{1}\right)\left(T_{1}-a_{2,2} I_{1}\right) \cdots\left(T_{1}-a_{n, n} I_{1}\right) .
$$

Suppose that, for an infinite number of integers $i$ there exists an integer $j$ such that the functional $\xi \rightarrow\left(S P_{i} \xi\right)_{j}$ is not continuous. Let the integers $i$ be $i_{1}, i_{2}, \ldots$ and the corresponding integers $j$ be $j_{1}, j_{2}, \ldots$ We have $j_{l}>i_{l}$ and, by transferring to a 
subsequence if necessary, we can assume $i_{l+1}>j_{l}$. Now define by induction a sequence of elements $\xi_{1}, \xi_{2}, \ldots$ of $E_{1}$, with

$$
\left\|\xi_{k}\right\|<2^{-k}\left\|\prod_{m=j}^{i_{k}}\left(T_{1}-a_{m, m} I_{1}\right)\right\|^{-1}
$$

for $j=1, \ldots, i_{k}-1$ and

$$
\left|\left(S P_{i_{k}} \xi_{k}\right)_{j_{k}}\right|>k\left\|\phi_{j_{k}}\right\|+\left|\sum_{l=1}^{k-1}\left(S P_{i_{l}} \xi_{l}\right)_{j_{k}}\right|
$$

where $\phi_{j}$ is the functional $\eta \rightarrow \eta_{j}$ in $E_{2}^{*}$. Put $\eta=\sum_{l=1}^{\infty} P_{i_{l}} \xi_{l}$. Then

$$
\begin{aligned}
(S \eta)_{j_{k}} & =\sum_{l=1}^{k}\left(S P_{i_{l}} \xi_{l}\right)_{j_{k}}+\left[S P_{j_{k}} \sum_{l=k+1}^{\infty} \prod_{m=j_{k}+1}^{m=i_{l}}\left(T_{1}-a_{m, m} I_{1}\right) \xi_{l}\right] j_{k} \\
& =\sum_{l=1}^{k}\left(S P_{i_{l}} \xi_{l}\right)_{j_{k}}
\end{aligned}
$$

so that $\left|(S \eta)_{j_{k}}\right|>k\left\|\phi_{j_{k}}\right\|$, which is impossible. There is thus an integer $p$ such that $\xi \rightarrow\left(S P_{i} \xi\right)_{j}$ is bounded for all $i \geqq p$ and all $j$.

Now let $\left\{x_{n}\right\}$ be a sequence in $E_{1}$ with $x_{n} \rightarrow 0$ and $S P_{p} x_{n} \rightarrow y$ in $E_{2}$. Since $y_{j}=\lim _{n}\left(S P_{p} x_{n}\right)_{j}=0$ for each $j, y=0$ and $S P_{p}$ is continuous by the closed graph theorem. Finally put

$$
Q=\left(T_{2}-a_{1,1} I_{2}\right) \cdots\left(T_{2}-a_{p, p} I_{2}\right) .
$$

Then $Q S=S P_{p}$ and so $Q S$ is continuous. Since the null space of $Q$ is void we can use 7.3 to show that $S$ is continuous.

NoTES. (i) The only possible elements of the point spectrum of an operator given by a triangular matrix are the entries on the diagonal.

(ii) If $E_{1}$ is a Fréchet space the above proof works providing we define the $\xi_{k}$ such that $\left\|P_{i_{k}} \xi_{k}\right\|_{n}<2^{-k}$ for $n=1, \ldots, k$ where \|\|$_{1},\|\|_{2}, \ldots$ is a sequence of seminorms on $E_{1}$ giving the metric topology.

(iii) The result and proof can be adapted to the case in which $E_{2}$ is an $F K$ space providing that there is a sequence $\left\{b_{n}\right\}$ of positive real numbers such that $\left|x_{n}\right|=o\left(b_{n}\right)$ as $n \rightarrow \infty$ for all $x$ in $E_{2}$. In this case we merely choose the $\xi_{l}$ so that we get

$$
\left|(S \eta)_{j_{k}}\right|>b_{j_{k}} \text {. }
$$

(iv) Any Fréchet space $F$ whose dual is separable in the $w^{*}$ topology is isomorphic with an $F K$ space since if $\phi_{1}, \phi_{2}, \ldots$ is a sequence in $F^{*}$ spanning it in the $w^{*}$ topology then $x \rightarrow\left\{\phi_{i}(x)\right\}$ maps $F$ one to one onto a sequence space which becomes an $F K$ space if we take it with the topology induced by this map.

9.2. Corollary. Let the conditions of 9.1 be satisfied except that instead of assuming that $\sigma_{p}\left(T_{2}\right)$ is void we assume that $\mathscr{R}\left(T_{1}-a_{i, i} I_{1}\right)$ is a closed subspace of $E_{1}$ of finite codimension for each $i$. Then $S$ is continuous. 
Proof. The proof is the same as in 9.1 except that we replace the last paragraph by the following.

The conditions now ensure that $\mathscr{R}\left(P_{p}\right)$ is a closed subspace of $E_{1}$ of finite codimension. Write $E_{1}$ as the direct sum of $R_{1}\left(=\mathscr{R}\left(P_{p}\right)\right)$ and a subspace $R_{2}$ of finite dimension. Since $P_{p}$ maps $E_{1}$ onto $R_{1}$ continuously the closed graph theorem implies that if $x_{n} \rightarrow 0$ in $R_{1}$ there exists a sequence $y_{n}$ in $E_{1}$ such that $y_{n} \rightarrow 0$ and $P_{p} y_{n}=x_{n}$. However $S P_{p}$ is continuous so that this implies $\lim _{n} S x_{n}=\lim _{n} S P_{p} y_{n}=0$ and thus $S$ is continuous on $R_{1} . S$ is also continuous on $R_{2}$ (a finite dimensional space) and hence $S$ is continuous on $R_{1} \oplus R_{2}=E_{1}$.

9.1 and 9.2 can be combined to give

9.3. THEOREM. Let the conditions of 9.1 be satisfied except that instead of assuming $\sigma_{p}\left(T_{2}\right)$ is void we assume that $\left\{T_{1}, T_{2}\right\}$ has no critical eigenvalues. Then $S$ is continuous.

Proof. We show that $S P_{p}$ is continuous as in Theorem 9.1.

Put

$$
\begin{aligned}
Q & =\left(T_{2}-b_{1} I_{2}\right) \cdots\left(T_{2}-b_{k} I_{2}\right), \\
P^{\prime} & =\left(T_{1}-c_{1} I_{1}\right) \cdots\left(T_{1}-c_{l} I_{1}\right),
\end{aligned}
$$

where $b_{1}, \ldots, b_{k}$ are those elements of $a_{1,1}, \ldots, a_{p, p}$ which are not in $\sigma_{p}\left(T_{2}\right)$ (repeated the same number of times) and $c_{1}, \ldots, c_{l}$ those which are. Then $S P_{p}$ $=Q S P^{\prime}$ and the method in 9.1 shows that $S P^{\prime}$ is continuous. Using the method of 9.2, which is applicable by virtue of 2.3 and 3.3, we see that $S$ is continuous.

This result is complete since 2.1 and 2.2 show that if $\left\{T_{1}, T_{2}\right\}$ has a critical eigenvalue then $S$ can be discontinuous. The results in this paper point to the truth of the following

9.4. Conjecture. Let $E_{1}, E_{2}$ be two Banach spaces, $T_{1}, T_{2}$ continuous linear operators in $E_{1}, E_{2}$ and $S$ a linear map of $E_{1}$ into $E_{2}$ with $S T_{1}=T_{2} S$. If $\left\{T_{1}, T_{2}\right\}$ has no critical eigenvalues then $S$ is continuous.

\section{REFERENCES}

1. N. Bourbaki, Éléments de mathématique, Espaces vectoriels topologiques, Chapters I-II, Actualités Sci. Ind. No. 1189, Hermann, Paris, 1953.

2. — Éléments de mathématique, Espaces vectoriels topologiques, Chapters III-V, Actualités Sci. Ind. No. 1225, Hermann, Paris, 1955.

3. N. Dunford, A survey of the theory of spectral operators, Bull. Amer. Math. Soc. 64 (1958), 217-274.

4. N. Dunford and J. T. Schwartz, Linear operators, Part II, Interscience, New York, 1963.

5. K. Hoffman, Banach spaces of analytic functions, Prentice-Hall, Englewood Cliffs, N. J., 1962.

6. B. E. Johnson, An introduction to the theory of centralisers, Proc. London Math. Soc. (3) 14 (1964), 299-320.

7. - Centralisers on certain topological algebras, J. London Math. Soc. 39 (1964), 603-614.

8. - Continuity of centralisers on certain Banach algebras, J. London Math. Soc. 41 (1966), 639-640. 
9. T. Kato, Perturbation theory for nullity, deficiency and other quantities of linear operators, J. Analyse Math. 6 (1958), 263-322.

10. L. H. Loomis, An introduction to abstract harmonic analysis, Van Nostrand, Princeton, N. J., 1953.

11. M. A. Naimark, Normed rings, Noordhoff, Groningen, 1959.

12. C. E. Rickart, General theory of Banach algebras, Van Nostrand, Princeton, N. J., 1960.

13. J. G. Wendel, Left centralizers and isomorphism of group algebras, Pacific J. Math. 2 (1952), 251-261.

14. J. H. Williamson, A theorem on algebras of measures on topological groups, Proc. Edinburgh Math. Soc. 11 (1959), 195-206.

15. K. Zeller, Theorie der limitierungsverfahren, Springer, Berlin, 1958.

THE UNIVERSITY,

NeWCASTle-UPON-TyNe, ENGLAND 